\title{
Revisión: ¿Puede el cambio climático afectar la producción de quinoa (Chenoponium quinoa willd.) en el Altiplano chileno?
}

\author{
Review: Can climate change affect production of quinoa (Chenopodium quinoa \\ willd.) crop on chilean Altiplano?
}

\author{
Matías Sánchez-Monje ${ }^{*}$, Jorge Olave ${ }^{2}$
}

\begin{abstract}
RESUMEN
El objetivo de esta revisión es dar cuenta de los principales avances en las investigaciones de los efectos del cambio climático en Sudamérica, cómo se abordan dichos estudios y cómo estos resultados pueden afectar al cultivo de la quinoa. Los principales efectos se pueden observar en la cuenca del Amazonas, donde se producirá un decrecimiento en las precipitaciones de 9 a $19 \%$ entre los años 2020 y 2080, lo que afectaría a cultivos como el maíz, disminuyendo su rendimiento en un 25\%. En el Altiplano habrá un cambio en el ciclo anual de las precipitaciones reduciéndose las lluvias tempranas (octubre - diciembre) e incrementándose las estivales (enero - marzo). En el caso de la temperatura también se prevé un incremento de 3 a $6{ }^{\circ} \mathrm{C}$, lo que tiene directa relación con la altitud por sobre los $3.500 \mathrm{msnm}$, ya que en zonas bajas no se presenta tal aumento. Los rendimientos en el cultivo de la quinoa podrán verse afectados por la disminución de las precipitaciones tempranas, pero podría haber efectos positivos debido a temperaturas más cálidas, reducción de las heladas y un aumento de la asimilación del carbono.
\end{abstract}

Palabras clave: quinoa, cambio climático, modelo de cultivo

\begin{abstract}
The objective of this review is to inform about the main advances in research on the effects of climate change in South America, how those studies are addressed and how those results can affect quinoa crop. The main effects can be observed in the Amazon basin where, between 2020 and 2080, a decrease in rainfall of 9 to 19\% would be observed, affecting crops such as maize, reducing its yield by $25 \%$. In the altiplano there will be a change in the annual cycle of precipitation, reducing early precipitation (October December) and increasing summer precipitation (January - March). In the case of temperatures also an increase of 3 to $6^{\circ} \mathrm{C}$ is foreseen which has a direct relation with the altitude above 3.500 masl since in low zones this increase is not shown. Yields in quinoa cultivation may be affected by the decrease in early precipitation, but positive effects could be seen due to warmer temperatures, frost reduction and increased carbon assimilation.
\end{abstract}

Keywords: quinoa, climate change, crop modelling

\section{Introducción}

La temperatura promedio de la Tierra y los océanos ha sido calculada mediante una tendencia lineal, lo que muestra un calentamiento de $0,85^{\circ} \mathrm{C}$ $\left(0,65\right.$ a $\left.1,06^{\circ} \mathrm{C}\right)$, en el periodo 1880 a 2012 , donde existe mucha información independiente (IPCC, 2014).
Este aumento en las temperaturas está preocupando a las autoridades en todo el mundo debido a las implicancias que tendría y sus posibles efectos sobre otras variables climáticas. Es por esto que el Panel Intergubernamental para el Cambio Climático (IPCC) se ha planteado diversos escenarios climáticos para estudiar de qué forma afectará a

1 Programa de Doctorado en Agricultura para Zonas Áridas - Desérticas. Facultad de Recursos Naturales Renovables. Universidad Arturo Prat. Iquique. Chile.

2 Centro de Investigación y Desarrollo de Recursos Hídricos (CIDERH). Universidad Arturo Prat. Iquique. Chile.

* Autor para correspondencia: matias.sanchez.monje@gmail.com

Fecha de Recepción: 03 noviembre, 2018.

Fecha de Aceptación: 12 diciembre, 2018.

DOI: http://dx.doi.org/10.4067/S0718-34292019005000202. Publicado en línea: 11-junio-2019. 
la Tierra en el futuro el aumento de la temperatura (IPCC, 2014).

El IPCC ha creado escenarios a partir de 1992, por ejemplo, cuando utilizó un conjunto de escenarios de emisiones como base para sus proyecciones climáticas, conocidos como escenarios IS92 (Nakicenovic and Stewart, 2000). El mismo año se publicaron nuevos escenarios de emisiones, los llamados Escenarios del IEEE (IPCC, 2000), más conocidos como escenarios SRES (Special Report on Emission Scenarios). Para el último reporte sobre cambio climático el IPCC estrenó los RCP (Representative Concentration Pathways) (IPCC, 2014), los cuales dan cuenta del forzamiento radiativo como factor a considerar en las proyecciones futuras.

Para relacionar los efectos que pueden tener los diferentes escenarios climáticos en la agricultura se ha complementado la información de los modelos climáticos con los modelos de cultivos. En la actualidad existen diversos modelos de cultivos con diferentes características. En la zona del Altiplano se han utilizado diferentes modelos, como el Aquacrop creado por FAO (Geerts, S., 2010), el cual se caracteriza por modelar cultivos bajo déficit hídrico. También está CropSyst, que usa módulos de crecimiento direccionados a la radiación y al agua (con 40 parámetros de cultivo). Otro modelo es WOFOST, basado en el consumo de carbono de las plantas (con 49 parámetros de cultivo) (Todorovic et al., 2009). El modelo SALTMED se caracteriza por modelar cultivos con déficit hídrico y estrés por salinidad, entre otros parámetros (Ragab et al., 2005). Un modelo de cultivo que destaca entre los demás es el SIMPROC (Santibáñez, 2001), ya que se caracteriza por realizar simulaciones de forma iterativa, para las fechas de siembra, lo que permite determinar la mejor fecha de siembra frente a escenarios cambiantes.

Como consecuencia del cambio climático se deben identificar especies que posean cualidades de tolerancia a estreses abióticos, de manera de poder asegurar la producción de alimentos en un futuro cambiante y con una población planetaria que sobrepase los nueve mil millones de personas en las décadas venideras. Una de las especies de cultivo que se cree puede ser un buen candidato para futuros escenarios de cambio climático es la quinoa (Chenopodium quinoa Willd) (Ruiz et al., 2014), la cual se adapta bien a suelos salinos, estrés hídrico y estrés por frío. ¿Pero son estas cualidades suficientes para considerar al cultivo de la quinoa un candidato confiable para producción de alimentos bajo escenarios de cambio climático?
Elobjetivode estarevisión es entregarinformación acerca de los últimos estudios sobre cambio climático en Sudamérica y en especial en la zona del Altiplano chileno-boliviano y cómo estas posibles condiciones futuras podrían afectar al cultivo de la quinoa.

\section{Materiales y métodos}

Se revisaron las fuentes bibliográficas contenidas en la Biblioteca de la Universidad Arturo Prat, Facultad de Recursos Naturales Renovables, además de diversos gestores de búsqueda bibliográfica como ScienceDirect, SciELO, Elsevier, Springerlink y Web of Science. Se utilizaron las palabras clave de búsqueda: quinoa, modelamiento, rendimiento, cambio climático, y se seleccionaron publicaciones en idioma español e inglés, de preferencia de los últimos 10 años, consideradas relevantes para la realización de la presente revisión bibliográfica.

\section{Resultados y discusión}

\section{Cambio climático}

Para el futuro se proyecta un aumento en la variabilidad climática, lo que impactará muy fuertemente en la producción alimentaria. Sin embargo, la magnitud y trayectoria de estos impactos son todavía inciertas, particularmente cuando las variables climáticas son menos predecibles. Hay presencia de eventos climáticos extremos con mayor frecuencia e intensos, por ejemplo, cuando las temperaturas exceden el rango óptimo para el crecimiento y desarrollo de los cultivos (Hannah et al., 2013). Para predecir estos cambios futuros en el clima existen los modelos de circulación global (MCG), los cuales son herramientas para el estudio de impactos potenciales bajo escenarios de posible cambio climático, donde se incluyen cambios en la temperatura, precipitación y nivel del mar. El Centro de Predicción del Clima perteneciente a la oficina de Administración Nacional del Océano y Atmósfera (NOAA) de Estados Unidos define el cambio climático como un "modelo matemático para describir cuantitativamente, simular y analizar la interacción entre la atmósfera y la superficie (ej. Océano, tierra y hielo)" (Schramm et al., 2014).

\section{Modelamiento del cambio climático}

Un MCG combina una serie de modelos de la atmósfera, océanos y superficie terrestre de la Tierra. 
Los modelos MCG dividen la Tierra en muchas capas y en miles de grillas tridimensionales. Estos modelos son específicos para replicar el clima pasado y presente. Por ejemplo, los MCG pueden reproducir con exactitud las tendencias de calentamiento temporal observado, dinámicas de hielo marino y los eventos climáticos extremos (IPCC, 2014).

Los MCG se usan para simular el clima presente y predecir climas futuros influenciados por gases de efecto invernadero y aerosoles. Desafortunadamente, están restringidos en su influencia para estudios locales debido a su resolución espacial (generalmente de alrededor de $50.000 \mathrm{~m}^{2}$ ) y no pueden resolver características a escala más pequeña como lo son las nubes o la topografía particular de una zona. Por lo tanto, las simulaciones de estos modelos son deficientes cuando el área de estudio tiene una topografía muy compleja (Wilby et al., 2002).

Cuando se requiere aumentar la resolución de los modelos climáticos para realizar estudios a nivel regional, se necesita disminuir la escala de la grilla (downscaling en inglés), para lo cual existen diferentes metodologías que se pueden agrupar en 4 tipos: a) modelamiento dinámico del clima, b) tipificación sinóptica del clima, c) generación estocástica del clima y d) enfoque basado en regresión (Wilby et al., 2002).

Disminuir la escala dinámicamente comprende la anidación de los modelos climáticos regionales (MCR) de alta resolución dentro de un MCG con una resolución más baja (Giorgi and Mearns, 1999).

Los MCR usan los MCG para definir cuánto varían las condiciones climáticas, donde las dinámicas físicas de la atmósfera son modeladas usando un espaciamiento horizontal de la grilla de 20 a $50 \mathrm{~km}$. La principal limitación de los MCR es su alta demanda computacional, al igual que los MCG. Los escenarios producidos por los MCR son también sensibles a la elección de los límites de las condiciones (como la humedad del suelo) usados en experimentos iniciales. La ventaja principal de los MCR es su capacidad para resolver características atmosféricas a una menor escala que los MCG. Además, los MCR pueden ser usados para explorar la significancia relativa de los diferentes forzamientos externos como lo son los ecosistemas terrestres o cambios atmosféricos químicos (Wilby et al., 2002).

\section{Cambio climático en Sudamérica}

En Sudamérica se han realizado diversas investigaciones para evaluar el estado de ciertos cultivos frente a escenarios de cambio climático. Es así como Jones y Thornton (2003) pudieron estimar que el rendimiento del cultivo de maíz decrecía en un 25\% en Brasil hacia el 2055, y que en Venezuela declinaba casi a 0 , lo que significa que se deben realizar grandes cambios en los sistemas productivos para que no ocurra migración de esas zonas agroproductivas.

En la cuenca del Amazonas y Sao Francisco se estiman disminuciones en las precipitaciones entre las décadas del 2020 a 2080 de un 9 a un 19\% y de un 15 a un 35\%, respectivamente (Marengo et al., 2012).

Urrutia y Vuille (2009) determinaron que las zonas montañosas tropicales de los Andes se verán más afectadas por cambios climáticos futuros que las zonas bajas aledañas. Las proyecciones indican un calentamiento significativo en los Andes tropicales, que es potenciado por la altura.

\section{Cambio climático en el Altiplano}

Análisis realizados con proyecciones del siglo 21 estiman que las precipitaciones promedio anuales en el Altiplano van a tener poca variación, pero habrá un cambio en el ciclo anual de las precipitaciones, reduciéndose las lluvias tempranas (octubre-diciembre) e incrementándose las estivales (enero - marzo) (Seth et al., 2010). Según Sarricolea and Aravena, 2015, en la comuna de Colchane, Región de Tarapacá de Chile, se prevé un incremento en las temperaturas que variará entre los 4 y $6{ }^{\circ} \mathrm{C}$, con un forzamiento radiativo de 8,5 $\mathrm{W} \mathrm{m}{ }^{-2}$. En otro estudio realizado por Urrutia y Vuille (2009), la simulación determinó un aumento de 3 ${ }^{\circ} \mathrm{C}$ para la zona del Altiplano en un escenario SRES A2 (altas emisiones de carbono) versus un escenario B2 (bajas emisiones de carbono). Bradley et al., (2006) encontraron también que las medias de las temperaturas en la zona de los Andes, sobre los 3.500 $\mathrm{msnm}$, serían mayores que las temperaturas medias a baja altura. Otros autores como Thibeault et al., (2010) observaron que las tendencias históricas hacia un alza en las temperaturas eran pocas si se comparaba 19812000 con 1960-1980. Sin embargo, si esta información se proyecta hacia el intervalo 2046-2065, el incremento de las temperaturas mínimas y máximas sería de 2,1 y $4,0^{\circ} \mathrm{C}$ y hacia el intervalo $2081-2100$ de 3,0 y 5,1 respectivamente. Según otros modelos de circulación global, la temperatura probablemente suba entre 1,3 a $1,6{ }^{\circ} \mathrm{C}$ para el año 2030 y entre 4,8 y $6,0^{\circ} \mathrm{C}$ para el año 2100 (Garreaud et al., 2009). 


\section{Modelamiento del cultivo de la quinoa}

Últimamente se han utilizado diversos modelos para conocer los rendimientos potenciales de la quinoa bajo diversos manejos de riego y escenarios climáticos. Los modelos pueden ser herramientas muy útiles en la gestión del agua para la agricultura. No sólo ayudan en la programación del riego y en la estimación de la demanda de agua por el cultivo, sino que también pueden ser utilizados para predecir los rendimientos y la salinización de los suelos (Ragab et al., 2005). El modelo SALTMED, por ejemplo, ha sido utilizado con éxito para simular la reducción del rendimiento de semillas y de materia seca en quinoa, por la salinidad del agua de riego (Razzaghi et al., 2011). En otra investigación, este modelo ha sido capaz de predecir la humedad del suelo, rendimiento total y materia seca para tres temporadas bajo diferentes estrategias de riego usando agua residual (Hirich et al., 2012).

Otro modelo que ha sido validado con relativo éxito para la quinoa es AquaCrop, que ha podido determinar el mejor manejo de riego deficitario para este cultivo en el Altiplano boliviano y cómo la disponibilidad de agua afecta al cultivo de quinoa (Geerts, S., 2010).

El modelo SIMPROC es otro acercamiento para modelar el cultivo de la quinoa. En particular tiene como base tres parámetros fundamentales: el índice de área foliar en conjunto con la intercepción de la radiación solar incidente, el cumplimiento de los requerimientos hídricos del cultivo y la temperatura (Santibáñez, 2001).

\section{Quinoa y cambio climático}

La quinoa posee una gran plasticidad y muchos la han nominado como un buen modelo para investigar los mecanismos que las plantas adoptan para enfrentar condiciones adversas, como la salinidad y sequía extrema (Ruiz et al., 2014). En este contexto, se puede mencionar la plasticidad fenotípica radicular de la quinoa, que le confiere una particular modificación anatómica que le permite una mayor capacidad de transporte para explorar las capas secas del suelo. Es así como la quinua no se ve afectada en su relación raíz/brote y en la longitud de raíz cuando existe un déficit de agua. También debido a su arquitectura radicular en forma de "espina de pescado" reduce la competencia entre raíces de la misma planta, y entre las raíces de plantas vecinas. La quinoa además presenta un sistema radicular con un eje principal robusto que le permite explorar capas profundas más rápidamente y más eficiente, fundamental para el éxito de esta planta en los primeros estadíos (Bazile, 2013).

Hay un escaso conocimiento de la respuesta que tendrá el cultivo de la quinoa frente a un cambio climático futuro. En este contexto, se ha simulado el rendimiento de la quinoa, y se ha encontrado un efecto positivo cuando la tendencia es hacia un clima más cálido, facilitando la asimilación de carbono y la reducción de la ocurrencia de heladas. Pero también se observa una disminución en la disponibilidad de agua, lo que provocará una merma del rendimiento, llegando en promedio a la mitad del potencial productivo del cultivo (Rambal et al., 2015).

\section{Conclusiones}

Si bien es cierto que la magnitud y trayectoria de los impactos del cambio climático son inciertas, se puede tener un acercamiento a lo que podría suceder dentro de 50 a 100 años mediante la aplicación de los MCG. Estos tienen robustez a escala global, pero cuando se requiere modelar a escala regional se necesita aumentar la resolución de la grilla, anidando el MCG con un MCR. Su principal limitante es la alta demanda en capacidad computacional, pero pueden resolver problemas a escala menor, como geografías irregulares.

El cambio climático en Sudamérica, principalmente, afectaría a la cuenca del Amazonas. En el Altiplano se prevé una variación en la estacionalidad de las precipitaciones que puede afectar al cultivo de la quinoa, por lo que se requerirá buscar variedades o ecotipos de ciclo corto y promover la adopción de tecnologías de riego más eficientes para paliar los efectos de la escasez hídrica en la época de siembra.

La información es escasa en lo que respecta a los efectos que provocaría un cambio climático en el norte de Chile y en especial en el Altiplano chileno y sur boliviano, y cómo afectaría a los cultivos de esta zona agroproductiva. Por ello es imperioso contar con una red de estaciones meteorológicas más robusta para la zona y realizar nuevas investigaciones debido a la importancia geopolítica y la riqueza en biodiversidad que poseen estos territorios. 


\section{Literatura citada}

Bradley, R.S.; Vuille, M.; Díaz, H.F.; Vergara, W. 2006. Threats to water supplies in the tropical Andes. Science, (80-. ). 312: 1755-1756.

Bazile, D.

2013. Estado del arte de la quinua en el mundo en 2013. FAO- CIRAD. Santiago, Chile- Montpellier, Francia. 724 p.

Garreaud, R.D.; Vuille, M.; Compagnucci, R.; Marengo, J. 2009. Present-day south american climate. Palaeogeogr Palaeoclim. Palaeoecol, 281: 180-195.

Geerts, S.; Raes, D.; García, G.

2010. Using aquacrop to derive deficit irrigation schedules. Agric. Water Manag., 98: 213-216.

Giorgi, F.; Mearns, L.O.

1999. Introduction to special section: Regional climate modeling revisited. J. Geophys. Res., 104: 6335-6352.

Hannah, L.; Roehrdanz, P.R.; Ikegami, M.; Shepard, A. V.; Shaw, M.R.; Tabor, G.; Zhi, L.; Marquet, P.A.; Hijmans, R.J. 2013. Climate change, wine, and conservation. Proc. Natl. Acad. Sci., 110: 6907-6912.

Hirich, A.; Choukr-Allah, R.; Ragab, R.; Jacobsen, S.-E.S.-E.;

El Youssfi, L.; El omari, H.

2012. The SALTMED model calibration and validation using field data from Morocco. J. Mater. Environ. Sci., 3: 342-359.

IPCC.

2014. Climate Change 2014: Mitigation of Climate Change: Contribution of Working Group III to the Fifth Assessment Report of the Intergovernmental Panel on. Cambridge Univ. IPCC. Press. $1132 \mathrm{p}$

2000. Informe especial del IPCC - escenarios de emisiones. Grup. Intergub. Expert. sobre el Cambio Climático 27p. https://doi.org/ISBN: 92-9169-413-4

Jones, P.G.; Thornton, P.K.

2003. The potential impacts of climate change on maize production in Africa and Latin America in 2055. Glob. Environ. Chang., 13: 51-59.

Klein, W.H.; Glahn, H.R.

1974. Forecasting local weather by means of model output statistics. Bull. Am. Meteorol. Soc. 55: 1217-1227.

Marengo, J.A.; Chou, S.C., Kay, G.; Alves, L.M.; Pesquero, J.F.; Soares, W.R.; Santos, D.C.; Lyra, A.A., Sueiro, G.; Betts, R.; Chagas, D.J.; Gomes, J.L.; Bustamante, J.F.; Tavares, P.

2012. Development of regional future climate change scenarios in South America using the Eta CPTEC/HadCM3 climate change projections: Climatology and regional analyses for the Amazon, São Francisco and the Paraná River basins. Clim. Dyn., 38: 1829-1848.

McGregor, J.J.

1997. Regional climate modelling. Meteorol. Atmos. Phys., 63: $105-117$.
Nakicenovic, N., Stewart, R.,

2000. Emissions Scenarios, Cambridge University Press. The Edinburgh Building Shaftesbury Road, Cambridge CB2 2RU, ENGLAND. https://doi.org/92-9169-113-5

Ragab, R.; Malash, N., Abdel Gawad, G.; Arslan, A.; Ghaibeh, A. 2005. A holistic generic integrated approach for irrigation, crop and field management: 1 . The SALTMED model and its calibration using field data from Egypt and Syria. Agric. Water Manag., 78: 67-88.

Rambal, S.; Ratte, J.P.; Mouillot, F.; Winkel, T.

2015. Trends in Quinoa Yield over the Southern Bolivian Altiplano: Lessons from Climate and Land-Use Projections. Quinoa Improv. Sustain. Prod., 47-62.

Razzaghi, F.; Plauborg, F.; Ahmadi, S.H.; Andersen, M.N.; Ragab, R. 2011. Simulation of Quinoa (Chenopodium Quinoa Willd.) Response To Soil Salinity Using the Saltmed Model. Vadose Zo., J. 25-32.

Ruiz, K.B.; Biondi, S., Oses, R.; Acuña-Rodríguez, I.S.; Antognoni, F., Martinez-Mosqueira, E.A., Coulibaly, A.; Canahua-Murillo, A.; Pinto, M.; Zurita-Silva, A.; Bazile, D.; Jacobsen, S.E., Molina-Montenegro, M.A.

2014. Quinoa biodiversity and sustainability for food security under climate change. A review. Agron. Sustain. Dev., 34: 349-359.

Santibáñez, F.,

2001. El Modelado del Crecimiento, Desarrollo y Producción de Maíz, sobre bases Ecofisiológicas, mediante el modelo SIMPROC. Rev. Agrometeorol. Argentina, 1:7-16.

Sarricolea, P.; Aravena, H.

2015. Variabilidad y cambios climáticos observados y esperados en el Altiplano del norte de Chile. Rev. Geogr. Norte Gd., 62: 169-183.

Schramm, P.J.; Uejio, C.K., Hess, J.J.; Marinucci, G.D.; Luber, G. 2014. Climate Models and the Use of Climate Projections: A Brief Overview for Health Departments. Atlanta, USA. 1-6pp.

Seth, A.; Rojas, M., Rauscher, S.A.

2010. CMIP3 projected changes in the annual cycle of the South American Monsoon. Clim. Change, 98: 331-357.

Thibeault, J.M.; Seth, A., García, M.

2010. Changing climate in the Bolivian Altiplano: CMIP3 projections for temperature and precipitation extremes. $J$. Geophys. Res. Atmos., 115: 1-18.

Urrutia, R.; Vuille, M.

2009. Climate change projections for the tropical Andes using a regional climate model: Temperature and precipitation simulations for the end of the 21st century. J. Geophys. Res. Atmos., 114: 1-15.

Wilby, R.L.; Dawson, C.W.; Barrow, E.M.; Wilby, R.L., Dawson, C.W.; Barrow, E.M.

2002. SDSM - a decision support tool for the assessment of regional climate change impacts. Environ. Model. Softw, 17: $147-159$. 
